\title{
Un estudio comparado de las reformas del sector seguridad y las relaciones civiles-militares en Perú, Chile, El Salvador y Guatemala ${ }^{1}$
}

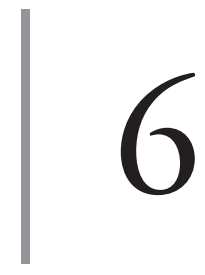

https://doi.org/10.21830/9789585350601.06

\author{
Martha Lucia Bahamón Jara \\ Ximena A. Cujabante Villamiß \\ Alex Camilo Durán Montaño ${ }^{4}$ \\ Universidad Militar Nueva Granada
}

\section{Resumen}

El capítulo presenta los resultados de una selección y estudio de cuatro casos de reforma del sector seguridad en América Latina, los cuales fueron seleccionados utilizando el criterio de diversidad y analizados a través de cuatro variables. El objetivo del análisis es hacer una caracterización exploratoria de los distintos procesos de reforma del sector seguridad en contextos de transición política en América Latina. El estudio arrojó los siguientes hallazgos clave: los obstáculos políticos, culturales y normativos al establecimiento del control democrático civil sobre las Fuerzas Armadas; las falencias que implica obviar reformas amplias que deben acompañar la transformación del sector seguridad en materia de justicia y desarrollo; las ventajas y desventajas de procesos de reforma al sector

1 Este capítulo es producto de investigación del proyecto INV-EES 2975 de 2019 "El panorama del post conflicto en Colombia: un acercamiento desde la gobernanza y la reforma del sector seguridad", financiado por la Universidad Militar Nueva Granada. Se reconoce la participación de la Universidad del Rosario en el marco del Convenio Especial de Cooperación con la Universidad Militar Nueva Granada.

2 Abogada de la Universidad Libre de Colombia, especialista en Derecho Administrativo de la Universidad Santo Tomás y magíster en Defensa de los Derechos Humanos y Derecho Internacional Humanitario. Exvicerrectora académica de la Universidad Militar Nueva Granada en el periodo 20092015. Directora de Posgrados de la Facultad de Relaciones Internacionales, Estrategia y Seguridad. Docente tiempo completo de carrera. Asesora y consultora de instituciones de educación superior. ORCID: https://orcid.org/0000-0002-5877-6886. Contacto: martha.bahamon@unimilitar.edu.co

3 Politóloga de la Universidad Javeriana, especialista en Negociación y Relaciones Internacionales de la Universidad de los Andes, magíster en Asuntos Internacionales y doctora en Estudios Políticos de la Universidad Externado de Colombia. Docente de tiempo completo de la Facultad de Relaciones Internacionales, Estrategia y Seguridad de la Universidad Militar Nueva Granada Orcid: https://orcid. org/0000-0002-5473-163X. Contacto: ximena.cujabante@unimilitar.edu.co

4 Politólogo de la Universidad de los Andes. Investigador de la Universidad Militar Nueva Granada. ORCID: https://orcid.org/0000-0001-9701-1520.Contacto: ac.duran1035@uniandes.edu.co 
seguridad inclusivos y, en contraposición, herméticos; y los peligros de tolerar prerrogativas económicas amplias para las Fuerzas Armadas y la corrupción endémica en sus filas y eslabones superiores. Por consiguiente, los tres casos analizados presentan una serie de lecciones y recomendaciones que deben ser atendidas en el actual proceso de reforma del sector seguridad que se busca en Colombia.

Palabras clave: Chile; El Salvador; Guatemala; Perú; reforma; seguridad.

\section{Introducción}

Para cumplir el objetivo de este capítulo es necesario analizar varios procesos de reforma del sector seguridad en América Latina. Mediante la metodología de estudio de caso y un criterio de diversidad, fueron seleccionados los siguientes: Perú, Chile, El Salvador y Guatemala. Los cuatro países ilustran la variedad de características que representan los factores detrás de procesos de reforma del sector seguridad en contextos de transición política. Un análisis profundo de estos cuatro casos permite caracterizar, de manera exploratoria, los distintos procesos de reforma del sector seguridad en contextos de transición política en América Latina.

Una de las variables básicas para evaluar los procesos de reforma del sector seguridad en los casos seleccionados es su grado de efectividad 5 . La siguiente variable, denominada control democrático civil, mide el grado en el que un proceso de reforma del sector seguridad instaura un sistema de relaciones cívico-militares que garanticen el control democrático de las Fuerzas Armadas. Mientras que las variables de efectividad y control democrático civil evalúan la medida en la que los procesos de reforma del sector seguridad en los casos seleccionados lograron o no la desmilitarización de la vida política, las siguientes dos variables evalúan la consecución o el rezago en el cumplimiento de los objetivos más amplios inherentes a un entendimiento más amplio de lo que es la gobernanza y la reforma del sector seguridad.

En primer lugar, la variable reformas de justicia y desarrollo analiza la medida en la que los procesos de reforma del sector seguridad en los casos

5 Según la Real Academia Espańola (RAE), la efectividad se puede entender como "la capacidad de lograr el efecto que se desea o se espera" (Real Academia Española, 2019). 
seleccionados han logrado o no cumplir objetivos más amplios en materia de desarrollo socioeconómico o acceso y administración efectiva de la justicia, concebidos como elementos claves de seguridad y justicia que sustentan la desmilitarización de las sociedades y el control democrático civil de las Fuerzas Armadas (Berg, 2012; Denney, 2013 ,2011; Hänggi \& Scherrer, 2008; Groner, 2012; Riley \& Schulz, 2016). En segundo lugar, el apoyo social y la apropiación local es la variable que mide el nivel de apoyo popular y de asociaciones positivas de la sociedad civil que logran los procesos de reforma del sector seguridad, lo cual es considerado crucial para que dichos procesos cuenten con suficiente caudal político para lograr de manera sostenible sus objetivos (Donais, 2009; Gordon, 2014; Heupel, 2012; Homel \& Masson, 2016; Oosterveld \& Galand, 2012).

Por último, mientras que la variable de economía política aborda uno de los principales desafíos detrás de la consolidación del control democrático de las Fuerzas Armadas ${ }^{6}$, la variable de la dimensión internacional analiza la injerencia de la sociedad internacional en los procesos de reforma del sector seguridad en los casos seleccionados. La ausencia o participación de organizaciones internacionales, sociedad civil internacional, agencias de la comunidad internacional del desarrollo y otros actores clave permite aseverar cuán hermético fue el proceso de reforma, así como generar conclusiones sobre el rol de estos actores en procesos endógenos de reforma del sector seguridad.

\section{Reforma del sector seguridad en Perú (1968-2001)}

Las Fuerzas Armadas han sido un actor político central en Perú durante casi más de un siglo. Antiguos oficiales militares han sido protagonistas electorales durante varias décadas (ganando varias contiendas en el proceso), y la premisa de que las Fuerzas Armadas peruanas tienen un papel legítimo en la gobernabilidad del país se ha aceptado en la vida política del país (Philip, 2013; Taylor, 2007). Este proceso se desarrolló fundamentalmente a partir del gobierno militar del general Juan Velasco Alvarado (1968-1980) y en los

6 De acuerdo con Fitch (1998). 
periodos políticos posteriores previos al ascenso de Alberto Fujimori a la presidencia (Burt, 2006; Comisión de la Verdad y Reconciliación [CvR], 2003; Graham, 1992; Philip, 2013; Walter, 2010).

Alberto Fujimori asumió la presidencia justificando soluciones drásticas a las múltiples crisis del país, condenando al establecimiento político, los sindicatos laborales y los grupos defensores de Derechos Humanos, y prometiendo restaurar el orden, eliminar la amenaza guerrillera y aniquilar la corrupción (Mauceri, 1995). A partir de la clausura del Congreso y la suspensión de la Constitución en 1992 con el apoyo de las Fuerzas Armadas ${ }^{7}$, el gobierno Fujimori inició un camino de recuperación económica y capturó algunos de los principales líderes de la insurgencia (Burt, 2006, p. 44), incluido el líder de Sendero Luminoso, Abimael Guzmán. En paralelo, el gobierno Fujimori inició una estrategia de neopopulismo, compuesta por un plan agresivo de gasto social y transferencias de fondos a organizaciones comunitarias (estrictamente controlado por el Ministerio de la Presidencia) (Roberts, 1995; Weyland, 1996).

A partir del autogolpe, el poder político de las fuerzas de seguridad fue extendido. Las autoridades civiles fueron suspendidas en zonas de emergencia, se instauraron leyes y decretos, y proliferaron las bases militares y programas de "acción cívica" para monitorear y controlar los movimientos de la población civil (Burt, 2006, pp. 41-42). Se fortaleció el Servicio Nacional de Inteligencia (SIN) y se desplegó el Grupo Colina ${ }^{8}$, de tal forma que el Sin, bajo la dirección de Vladimiro Montesinos, se convirtió en un instrumento de policía política del régimen usado para intimidar y silenciar críticos del régimen a partir de actos violentos, tales como la masacre de Barrios Altos ${ }^{9}$ o la masacre de Cantuta $^{10}$ (Asociación pro-Derechos Humanos, 1994; Burt, 2006, p. 47).

El poder político de las fuerzas de seguridad se conjugó con un menor control de su accionar por parte de las autoridades democráticas. Hubo un

7 Periodo de crisis constitucional conocido como el autogolpe o Fujigolpe.

8 Grupo paramilitar bajo la autoridad del Sin y el Servicio de Inteligencia del Ejército (SIE) (CVR, 2003).

9 Masacre ocurrida en Lima en 1991, donde una unidad del Grupo Colina asesinó 15 personas e hirió cuatro más sin conexiones políticas con el pretexto de asesinar miembros presuntos de Sendero Luminoso (Aprodeh, 1994).

10 Desaparición forzosa y asesinato de nueve estudiantes y un profesor de la Universidad de Cantuta en 1992 (Cvr, 2003). 
mayor uso de la represión legal, a través de la encarcelación indiscriminada de sospechosos de subversión y legislación "antiterrorismo" (CvR, 2003; Degregori \& Rivera, 1993). Las cortes militares usualmente reclamaban la jurisdicción sobre casos de supuestas violaciones de Derechos Humanos por parte de agentes del Estado y, amparados en la Ley de Amnistía de 1995, suministraban la salvaguarda institucional para que se siguieran violando Derechos Humanos impunemente (Burt, 2006, p. 51). Al cierre del siglo XX, las fuerzas de seguridad en Perú se convirtieron en instrumentos de represión política y diseminación del miedo, a servicio del régimen Fujimori.

El proceso electoral del año 2000 impulsó mayor oposición doméstica e internacional al régimen Fujimori, en la medida en que las condiciones que justificaron los abusos de poder del régimen cambiaron. Principalmente, la lucha contrainsurgente usada para justificar los abusos de poder ya no era perentoria por la virtual derrota militar de Sendero Luminoso, el apoyo público para el régimen disminuyó considerablemente y segmentos de la élite económica expresaron temor y desaprobación a las prácticas autoritarias y corruptas del régimen (Burt, 2006, pp. 56-57). Aunque Alberto Fujimori asumió un tercer mandato en medio de protestas sociales multitudinarias, la publicación de varias cintas de video mostrando a Montesinos sobornando a un congresista opositor precipitó el fin del régimen en noviembre del año 2000 y, luego de la conformación de un gobierno interino a cargo de Valentín Paniagua Corazao, la elección de Alejandro Toledo como presidente en 2001. Buscando responder al desafío de restaurar la confianza pública en las instituciones estatales, el gobierno Toledo (2001-2006) se comprometió a realizar un proceso de reforma del sector seguridad. Desde su discurso de posesión en 2001, Toledo anunció reformas en el sector militar y policial (Costa \& Nield, 2007, p. 115).

En cuanto a efectividad, la transformación de la Policía Nacional peruana fue uno de los elementos centrales del proceso de reforma del gobierno Toledo. Aunque en su discurso inicial Toledo anunció que la reforma policial sería una prioridad política, fue ambiguo sobre cómo lograrla. Su partido no había formulado propuestas específicas sobre cómo abordar la reforma policial en un contexto electoral en el cual el tema había sido poco discutido, y la naturaleza repentina de la transición política peruana — colapso súbito del gobierno- 
no permitió un margen suficiente para discutir y crear consensos alrededor de reformas institucionales (Costa, 2006, p. 216).

Por consiguiente, para identificar el efecto deseado o esperado de la reforma policial, es importante analizar el documento titulado "Fundamentos de la Reforma Policial”, escrito el 27 de julio de 2001 (en la víspera de la posesión de Alejandro Toledo como presidente) por el equipo reformador del Ministerio del Interior, compuesto por el ministro Fernando Rospigliosi —un periodista destacado especializado en relaciones cívico-militares y asuntos de inteligencia_-, el viceministro Gino Costa — jefe de la unidad de reforma de la Organización de Naciones Unidas (ONU) en El Salvador y que luego trabajó en la Oficina de Derechos Humanos de la Defensoría del Pueblo del Perú-y los asesores Carlos Basombrío y Susana Villarán ${ }^{11}$. El documento elaborado por este equipo estableció, como objetivo principal de la reforma policial, la recuperación de la confianza pública en la Policía Nacional del Perú (PNP) y su legitimidad (Costa, 2006, p. 219). A partir de este objetivo inicial, el documento aduce que era necesaria una evaluación adicional de la labor policial en Perú para saber qué partes de la PNP funcionaban bien y cuáles no, y que dicho proceso debía ser inclusivo e incorporar las perspectivas de múltiples actores relevantes con el fin de familiarizar el personal civil con el estado actual de la labor policial en Perú (Costa, 2006, p. 218).

Además de la reforma policial, también se emprendieron procesos de reforma en las Fuerzas Armadas, los servicios de inteligencia y el sector judicial. En respuesta al proceso de reforma policial en octubre de 2001, el Ministerio de Defensa peruano creó una comisión para evaluar las Fuerzas Armadas y recomendar medidas pertinentes de reforma, proceso en el cual se propuso una nueva Ley de Ministerio de Defensa para fomentar el liderazgo civil de las fuerzas a través de un nuevo ministerio con un carácter más civil a través de la incorporación de personal y una nueva estructura administrativa (Costa, 2006, p. 221). Simultáneamente, se buscó una modificación del enfoque misional del Ejército peruano para combatir los remanentes de Sendero Luminoso en áreas

11 Carlos Basombrío fue el exdirector del Instituto de Defensa Legal (una de las ONG peruanas más influyentes en materia de defensa de los Derechos Humanos), mientras que Susana Villarán fue ministra de la Mujer y Poblaciones Vulnerables en el gobierno de transición de Paniagua, y directora de una coalición de algunas ONG para la defensa de los Derechos Humanos. 
apartadas del país con estándares más altos de profesionalización, legitimidad pública y costo-eficiencia o maximización de recursos, con una autonomía reducida y mayor rendición de cuentas frente a autoridades civiles (Jaskoski, 2013, pp. 2-11).

En materia de reforma de los servicios de inteligencia, el propósito fue crear una nueva agencia por completo. Poco tiempo después del colapso del régimen Fujimori, el nuevo gobierno de Paniagua decretó la Ley 27351, que desactivó el Servicio de Inteligencia Nacional (SIN). Posteriormente, la intención ostensible fue crear una nueva agencia de inteligencia que reflejara los principios democráticos de la era post-Fujimori (Weeks, 2008, p. 54). Con ese espíritu, en julio de 2001, la Ley 27479 creó el Consejo Nacional de Inteligencia ( $\mathrm{CNI})$.

Si se entiende la efectividad como la capacidad de lograr el efecto que se desea o se espera (Real Academia Española, 2019), es posible aseverar que la efectividad del proceso de reforma del sector seguridad en Perú es cuestionable. Como se explicará a continuación, la reforma policial en Perú no se concretó ni tuvo sostenimiento porque las resistencias y la falta de apoyo presidencial socavaron el ímpetu del equipo reformador al seno del Ministerio de Defensa. Asimismo, la reforma de las Fuerzas Armadas no se tradujo en el enfoque misional contrainsurgente con mayores estándares de profesionalización, legitimidad pública y maximización de recursos. Además, el CNI mantuvo la opacidad del Sin y la resistencia de las Fuerzas Militares que habían monopolizado la labor de inteligencia no permitió mayor control civil. Por último, y de manera más amplia, el proceso de reforma del sector seguridad peruano evidenció vacíos en materia de reformas integrales orientadas a justicia y desarrollo, como también una apertura del proceso a mayor participación de actores internacionales.

En relación con el control democrático civil, el proceso de reforma de la PNP no logró que esta institución recuperara la confianza pública ni su legitimidad, principalmente debido a la resistencia interna por parte de la Policía y la falta de apoyo y liderazgo del gobierno Toledo. La resistencia dentro de la institución policial provenía de tres fuentes distintas, a saber: 
Los oficiales comunes y corrientes, que temían el cambio y se sentían amenazados por las nuevas reglas de juego [...] los oficiales tradicionales o institucionalistas — sobre todo oficiales generales y superiores, muchos de ellos retirados - a quienes les preocupaba que el proceso de desmilitarización diera pie a la indisciplina, fuera acompañado por la pérdida de los beneficios que la homologación con las Fuerzas Armadas había permitido [...]. Y por último, la de quienes protegían intereses creados, las más veces vinculados al manejo corrupto de los recursos institucionales. (Costa \& Basombrío, 2005, p. 82)

Por otro lado, la falta de apoyo del gobierno Toledo fue producto de la misma fragilidad del gobierno democrático. Los bajos niveles de aprobación presidencial y el constante prospecto de caída del nuevo régimen democrático envolvieron al gobierno Toledo en una atmósfera de inestabilidad política (Costa \& Basombrío, 2005, p. 83). La debilidad del gobierno y su falta de apoyo frente al proceso de reforma policial también condujo a que otras instituciones públicas, incluidos los partidos políticos, no apoyaran los esfuerzos de reforma realizados por el Ministerio del Interior (Costa, 2006, p. 227). Aunque la falta de involucramiento del gobierno tuvo ventajas, como la autonomía del equipo reformador y la celeridad de reformas que dependían netamente de la voluntad y autoridad del equipo del Ministerio del Interior, hubo múltiples asuntos pendientes y la salida de este equipo en enero de 2003 socavó cualquier apoyo desde las autoridades civiles al proceso de reforma policial.

Frente a reformas de justicia y desarrollo, se encuentra que el gobierno Toledo tampoco logró avanzar reformas en el sector de justicia. En materia de justicia, el gobierno Toledo paralizó el proceso de transferencia de los penales controlados por la PNP al Instituto Nacional Penitenciario (INPE). Este proceso, clave para fomentar la administración efectiva de justicia en el país y reducir la percepción de impunidad, fue detenido con el argumento de que el INPE carecía del personal necesario para asumir las funciones que le correspondían de acuerdo con la ley (Costa \& Basombrío, 2005, p. 83). El proceso de reforma del sector seguridad, particularmente de reforma policial, no tuvo un impacto significativo en la cantidad de delitos reportados; no hubo un aumento significativo en los niveles de denuncia (Basombrío, 2004, pp. 113-114). Estos datos indican que la falta de reformas más amplias en el sector justicia se tradujeron a una falta de confianza persistente de la ciudadanía en la administración efectiva de justicia y judicialización oportuna de delitos. 
En cuanto a apoyo social y apropiación local, a pesar de sus falencias en relación con las otras variables, el proceso de reforma del sector seguridad en Perú intentó generar mayor apoyo social y apropiación local a partir de la comunicación. Los logros alcanzados durante el proceso de reforma policial fueron publicitados y ofrecieron a la ciudadanía una visión positiva del manejo y la gestión de políticas policiales (Costa \& Nield, 2007 p. 124). Esto constituye una lección positiva para otros procesos de reforma, en la medida en que la transparencia, la comunicación y la entrega oportuna de información sobre políticas y procesos son centrales para crear confianza y diálogo con la sociedad civil, los cuales son necesarios para generar una masa crítica y, eventualmente, apoyo social masivo a los procesos de reforma.

Sin embargo, los avances del proceso de reforma del sector seguridad en Perú en materia de comunicación fueron opacados por las falencias en materia de inclusión. En primer lugar, el proceso de reforma policial fue liderado por un núcleo de civiles reformistas en el Ministerio del Interior sin constituir alianzas más amplias con organizaciones de la sociedad civil, salvo un grupo selecto de ONG (Costa, 2006, p. 226). En segundo lugar, estas autoridades civiles no establecieron coaliciones efectivas dentro de la Fuerza Pública. Por un lado, esto causó que las iniciativas dependientes del comando policial progresaran de manera lenta o nunca se materializaran, por ejemplo, en lo relacionado con la reforma de la adquisición de bienes y servicios y la modernización de sistemas de gestión (Costa, 2006, p. 226). Por otro lado, las Fuerzas Militares fueron reticentes en cooperar con el Ministerio del Interior, como también en asistir a la Policía durante la crisis de orden público, producto del recelo que generaba la percepción de creciente influencia política de la Policía (Costa, 2006, p. 227). Por consiguiente, el carácter hermético del proceso de reforma causó la inhabilidad de crear coaliciones críticas para llevar adelante los procesos de reforma de la Policía y las Fuerzas Militares.

La exclusión de la sociedad civil (entre otros sectores) se aunó al contexto de seguridad de Perú en el momento para crear condiciones en las cuales se resquebrajó la autonomía militar, pero no se tradujo en mayor legitimidad institucional de las Fuerzas Militares. Si bien las Fuerzas Militares perdieron influencia política en la transición democrática por la derogación del servicio 
militar obligatorio y la resolución de conflictos limítrofes con Ecuador y Chile, al mantenerse el miedo de la ciudadanía frente a un posible resurgimiento de Sendero Luminoso, las autoridades civiles no cambiaron el paradigma de control territorial y combate de amenazas a la soberanía que guio la operación de las fuerzas en el territorio peruano (Costa, 2006, p. 227; Jaskoski, 2013, p. 3). Puesto que aún se priorizaron el control territorial y las intervenciones militares aisladas, la creación de medidas de construcción de confianza con las comunidades o la instrumentalización del uso de la fuerza como parte de una oferta institucional económica y social más amplia no fueron prioridad. Así, las Fuerzas Militares en Perú no aumentaron de manera considerable su legitimidad pública (Jaskoski, 2013).

Haciendo hincapié sobre la economía política, el proceso de reforma del sector seguridad en Perú dejó pendiente la lucha contra la corrupción; persistieron condiciones de corrupción y malversación de fondos en la Policía y las Fuerzas Militares. Uno de los casos más notorios de corrupción fue la licitación de combustibles de la PNP. A pesar de abogar por la implementación de un sistema de adquisición y distribución de combustibles que generara mayor ahorro al erario público y menos irregularidades, el proceso no se reformó por las resistencias dentro de la fuerza (Costa \& Basombrío, 2005, p. 81). De igual forma, el sistema de adquisición de medicinas de la Fuerza Pública siguió plagado de irregularidades y carente de los controles necesarios (Costa \& Basombrío, 2005, p. 81). Los casos abundan porque la corrupción y malversación de fondos siguieron siendo comportamientos ampliamente aceptados en las instituciones del sector seguridad.

Por último, teniendo en cuenta la dimensión internacional, el proceso de reforma del sector seguridad en Perú se caracterizó por su hermetismo, pues no tuvo incidencia de organizaciones internacionales. Aunque la Comisión de Verdad y Reconciliación (CvR) fue una de las instituciones más emblemáticas producto de este proceso y de la transición a la democracia en Perú, la Cvr tampoco tuvo incidencia de garantes o cooperantes internacionales. En la Cvr participaron las Fuerzas Armadas, integrantes de Sendero Luminoso, el Movimiento Revolucionario Tupac Amarú (MrTA) y algunos funcionarios de los gobiernos de Alan García y Alberto Fujimori, pero no hubo participación 
ni transferencia de conocimiento notable de expertos o aliados internacionales (Cvr, 2003). Esta ausencia de la participación activa de referentes o expertos internacionales también aplica a los procesos de reforma de la Policía y las Fuerzas Militares peruanas.

\section{Reforma del sector seguridad en Chile (1969-1990)}

Las Fuerzas Armadas chilenas, a diferencia de su institución homóloga en Perú, recientemente se constituyeron como un actor político central en el país. Antes del golpe de Estado de 1973, las Fuerzas Militares de Chile habían emprendido un proceso de despolitización durante aproximadamente cinco décadas, que llegó a un súbito fin en el marco de sucesivos derrocamientos de gobiernos civiles por parte de las Fuerzas Militares. Una proporción importante de los militares pasaron de ocupar puestos gubernamentales a retraerse significativamente de la vida política, inclusive creando grupos endogámicos de oficiales (Sagredo \& Gazmuri, 2005). Sin embargo, en 1969 hubo una repolitización de algunos elementos de las Fuerzas Militares al participar en el Tacnazo, el cual constituyó una protesta de las Fuerzas ante el gobierno de Eduardo Frei Montalva (1964-1970) por falta de fondos (González, 2013, p. 28). Posteriormente, en 1970, Salvador Allende ascendió a la presidencia ${ }^{12}$.

La presidencia de Salvador Allende (1970-1973) fue un periodo convulsionado en la historia económica y política de Chile (Collier \& Sater, 1996; Nove, 1986). A pesar del declive económico, la coalición del presidente Allende consolidó su poder legislativo en las elecciones parlamentarias de marzo de 1973 y, en respuesta, la Democracia Cristiana y el Partido Nacional configuraron la Confederación de la Democracia (CODE). El estancamiento entre la Unión Popular de Allende y la CODE creó una parálisis legislativa que impidió el pasaje de iniciativas ejecutivas (Petras \& Morley, 1974).

12 Allende recibió el número más alto de votos en las elecciones presidenciales chilenas, pero al no ganar por una mayoría absoluta, el Congreso Nacional tuvo la potestad de elegir el presidente entre los candidatos. Allende recibió 36,6 \% del voto, mientras que su principal contrincante, Jorge Alessandri Rodríguez, recibió 35,3 \% y Radomiro Tomic tuvo 28,1 \% en tercer lugar (Jobet, 1987). Luego del anuncio de Alessandri afirmando que renunciaría si el Congreso Nacional lo eligiera, el Congreso eligió a Allende. 
Allende intentó ofrecer prerrogativas a técnicos de la industria del cobre para permanecer en Chile y la venta de cobre por divisas, como también mantener una relación de trabajo con las Fuerzas Militares chilenas hasta que milicias locales pudieran establecerse y consolidarse (Petras \& Morley, 1974). Estas iniciativas unilaterales del gobierno Allende y el continuo declive económico precipitaron una crisis de gobernabilidad (Petras \& Morley, 1974).

La crisis de gobernabilidad se agudizó a finales de agosto de 1973 (Bawden, 2016), y el 11 de septiembre de 1973, luego de la captura de Valparaíso por parte de la Armada chilena y el bombardeo y posterior toma del palacio presidencial de La Moneda, el gobierno Allende fue derrocado y el entonces comandante-en-jefe del Ejército chileno, Augusto Pinochet Ugarte, asumió el cargo de presidente. El golpe militar de 1973 le ofreció a las Fuerzas Militares la oportunidad para materializar las reivindicaciones expuestas en el Tacnazo y salir del ostracismo político.

Durante los primeros años del régimen militar, las Fuerzas Militares adoptaron una conducción unitaria, en la medida en que el Ejército logró reafirmar su primacía sobre el resto de las ramas de las Fuerzas y desempeñar de mejor forma las funciones represivas iniciales (Varas, 1981, p. 4). De igual forma, el contexto económico convulsionado y posterior recesión durante 1975 y 1976 afianzó aún más la consolidación del mando institucional de las Fuerzas Armadas y su definición como el soporte institucional del régimen. Sin embargo, en el contexto de esa crisis política y económica nacional más amplia, existió una creciente tensión entre sectores empresariales que querían transferir el poder político al capital privado y los grupos que querían retener el control estatal del país (Varas, 1981, p. 20). Las Fuerzas Armadas, por su tendencia estatista reticente a amenazar su autonomía corporativa con nuevas formas de control civil, buscaron asegurar la potestad de ejercer un papel político en términos institucionales (Varas, 1981, p. 21).

Estas tensiones desembocaron en la Constitución de 1980. Establecer una nueva Constitución era un asunto clave para el régimen militar, ya que representaba mitigar las tensiones previamente descritas y legitimar su dirección del país; para este propósito el régimen militar reunió un grupo de civiles "notables" para conformar una comisión responsable de redactar la nueva carta 
magna (Allan \& Pollack, 1990). La nueva Constitución de Chile fue aprobada a través de un plebiscito nacional celebrado el 11 de septiembre de 1980, y entró en vigencia el 11 de marzo del siguiente año. Esta Constitución le dio a las Fuerzas Armadas la posibilidad de ejercer un papel político activo en términos institucionales ${ }^{13}$.

Comprendido dentro del pasaje de la Constitución de 1980 se programó la celebración de un plebiscito en octubre de 1988 para votar sobre un nuevo término presidencial de ocho años para Pinochet, y el 5 de octubre de 1988 el régimen militar perdió el plebiscito (Roberts, 1995). Aunque Pinochet permaneció como presidente de Chile durante un año más como parte de las provisiones constitucionales, el siguiente año se transfirió el poder a Patricio Aylwin por su victoria en las elecciones generales de 1989. No obstante, Pinochet permaneció siendo comandante-en-jefe del Ejército hasta marzo de 1998 (Bawden, 2016).

Aunque nominalmente las Fuerzas Militares se retiraron de la vida pública como resultado de las elecciones generales de 1989, la transición chilena a la democracia fue pactada, entre el gobierno militar y la oposición política al régimen militar. Hubo un pacto tácito que involucró la aceptación por parte de la oposición de la inclusión de las Fuerzas Armadas en el proceso político (Godoy, 1999, p. 79). De igual forma, la oposición política aceptó el fuero parlamentario de Augusto Pinochet como senador vitalicio (Godoy, 1999, p. 79). La reforma a la Constitución de 1980 aprobó lo que se había negociado, como también aquello que pasó inadvertido en las negociaciones.

$\mathrm{Al}$ discutir la efectividad del proceso de reforma del sector seguridad en Chile, cabe acotar que el objetivo general de dicho proceso era erosionar el poder político de las Fuerzas Militares, particularmente a partir de desmontar las salvaguardas institucionales que el régimen militar de Augusto Pinochet había erigido. El régimen militar logró establecer una derecha política fuerte y

13 En primer lugar, los comandantes en jefe debían ser nombrados por el presidente entre los cinco oficiales de mayor antigüedad por periodos de cuatro años, y solo podían ser removidos de acuerdo con un Consejo de Seguridad Nacional donde dichos oficiales ejercían una mayoría (Varas, 1981, p. 21). Por otro lado, al Consejo de Seguridad Nacional le fue otorgada la facultad de nombrar dos miembros del Tribunal Constitucional, dictar su propio reglamento y presentar su opinión ante cualquier autoridad establecida por la Constitución (Varas, 1981, p. 21). 
unas Fuerzas Militares que comandaban un poder político amplio a través de la Constitución de 1980 y la Ley Orgánica de las Fuerzas Armadas de 1989, con lo cual creó una simbiosis entre fuerzas electorales conservadoras y las prerrogativas de las Fuerzas Armadas en la transición democrática (Ensalaco, 1994; Geisse \& Ramírez, 1989). Si se juzga el proceso de reforma del sector seguridad en Chile con este rasero, se pueden hablar de victorias tempranas y logros graduales pero resultados limitados.

Por otro lado, un objetivo importante del proceso de reforma del sector seguridad era investigar las desapariciones después de arrestos, torturas o ejecuciones cometidas por agentes del gobierno, así como los asesinatos y amenazas a la vida de los ciudadanos por motivos políticos (Hilbink, 2007). Para este propósito, se estableció la Comisión Nacional de la Verdad y la Reconciliación, también conocida como la Comisión Rettig. Aunque no existió un proceso amplio de reforma a la justicia, la Comisión Rettig logró unos resultados notables en materia de establecimiento de la verdad y recomendaciones para la no-repetición, lo cual atestigua cierta efectividad del proceso en materia de reforma judicial.

En lo relacionado con el control democrático civil, el régimen militar exhibió una previsión considerable al instrumentalizar la Constitución de 1980 y la Ley Orgánica de las Fuerzas Armadas de 1989 para asegurar ventajas para fuerzas electorales conservadoras y prerrogativas políticas para las Fuerzas Armadas. Estas salvaguardas institucionales probarían reducir notablemente los prospectos de control democrático civil de las Fuerzas Armadas en la transición a la democracia. De igual forma, las Fuerzas Armadas lograron preservar prerrogativas que les daban poder de veto sobre políticas a nivel nacional y blindarse de menor autonomía o mayor responsabilidad vis-á-vis las autoridades políticas civiles. A pesar de algunas reformas tempranas en la presidencia de Patricio Aylwin, las reformas políticas sustanciales para reducir la autonomía militar se verían frenadas.

En primer lugar, las provisiones para curules designadas en el Senado y el sistema electoral binomial beneficiaron la derecha política y las Fuerzas Militares. En el Senado, nueve curules estaban reservadas para oficiales no-electos seleccionados por el régimen militar saliente y, como consecuencia, 
más de un tercio de las curules del Senado serían ocupadas por políticos simpatizantes de las Fuerzas Armadas (Hunter, 1997, p. 456). De igual forma, el sistema binomial mayoritario, que asigna dos escaños por distrito y le otorga mayoría a un partido o alianza que controle poco más de un tercio del voto y la mitad de los escaños, terminó garantizando que los parlamentarios afines al régimen militar tuvieran una influencia legislativa desproporcionada frente a su presencia en el Congreso (Hunter, 1996, p. 456).

El Consejo de Seguridad Nacional fue uno de los vehículos institucionales mediante los cuales las Fuerzas Armadas limitaron el control democrático civil sobre su accionar y centralidad política. El Consejo, establecido durante la transición a la democracia, tenía poder de veto sobre múltiples políticas nacionales y potenció la autonomía de las Fuerzas Armadas, en la medida en que se limitó la habilidad del presidente de despedir a los comandantes del Ejército, Armada, Fuerza Aérea y Carabineros (límite que permaneció durante la investidura de Pinochet como comandante del Ejército hasta 1998) (Atria, 1994; Hilbink, 2007).

Sin embargo, se utilizaron otros vehículos de orden institucional y procedimental para limitar el control democrático civil de las Fuerzas Armadas en la transición chilena a la democracia. Primero, se establecieron barreras procedimentales a cualquier reforma o enmienda constitucional, confiriéndoles garantías constitucionales a las Fuerzas Armadas. Aunque inicialmente la Concertación de Partidos por la Democracia logró aprobar reformas para que los miembros designados del Senado tuvieran menos gravitas y para eliminar el papel mayoritario de las Fuerzas Armadas en el Consejo de Seguridad Nacional, además de lograr un quórum menor para reformas constitucionales, las salvaguardas institucionales ya logradas por el régimen militar y sus aliados políticos obligaron a la Concertación a acudir a apoyo político de algunos segmentos de la derecha para implementar políticas, y así se bloquearon mayores esfuerzos por reformar el sistema político (Hunter, 1997, p. 457).

A pesar de la escasez de reformas constitucionales, sí se lograron algunas afirmaciones de control democrático civil sobre las Fuerzas Armadas en la presidencia de Patricio Aylwin (1990-1994), aunque los esfuerzos de reforma de su sucesor no fueron exitosos. El presidente Aylwin usó su autoridad como comandante-en-jefe para rechazar algunas propuestas de Pinochet en 
materia de ascensos en la fuerza, se diluyó el papel de las Fuerzas Militares en labores de orden público por la devolución de la responsabilidad política de los Carabineros al Ministerio del Interior y la exclusión de las Fuerzas Militares de la Oficina Coordinadora de Seguridad Pública (Fuentes, 2002). En octubre de 1995, el gobierno de Eduardo Frei Ruz-Tagle (1994-2000) sometió al Congreso un núcleo de enmiendas constitucionales, incluyendo propuestas para eliminar la designación de senadores, reestructurar el Consejo de Seguridad Nacional para permitir mayor autoridad civil, y la investidura de mayores poderes de supervisión de las Fuerzas Armadas al Congreso; estos esfuerzos fueron bloqueados en mayo de 1996 (Weyland, 1997).

En materia de reformas de justicia y desarrollo, la reforma del sector justicia era uno de los asuntos neurálgicos del proceso de reforma del sector seguridad en Chile. Mientras que para las Fuerzas Armadas tener que aceptar responsabilidad por vejámenes cometidos tocaba un nervio sensible y varios oficiales estaban dispuestos a resistir el procesamiento judicial acarreando un gran costo político, para aquellos con familiares y seres queridos desaparecidos la justicia era imprescindible en cualquier esfuerzo por transformar positivamente las relaciones cívico-militares (Hunter, 1997, p. 460). Por consiguiente, los partidos de la Concertación emprendieron un curso de acción cauto, reforzando los llamados a la justicia sin poner en peligro la consolidación democrática y la estabilidad por medio de un desafío directo a las Fuerzas Armadas; el caso chileno sigue siendo conocido por el poco éxito de los miembros de la justicia en perseguir y enjuiciar responsables de violaciones de Derechos Humanos (Hunter, 1997, p. 460).

Aunque el gobierno Aylwin obedeció un mandato popular amplio al establecer la Comisión Rettig, esta incorporó elementos que coadyuvaron la impunidad, y el gobierno buscó preservar la connivencia entre la rama judicial y el establecimiento militar; ambas instituciones fueron aliadas cercanas durante el régimen militar. En primer lugar, a la Comisión se le encargó recopilar un récord de las víctimas de violaciones de Derechos Humanos durante el régimen militar, mas no de los victimarios (Comisión Nacional de Verdad y Reconciliación, 1991). De igual forma, aunque el gobierno Aylwin instó a las familias de las víctimas a utilizar el sistema judicial para enjuiciar a sus victima- 
rios con información recopilada por la Comisión Rettig, el gobierno mantuvo la amnistía general ofrecida a los abusos de Derechos Humanos cometidos entre 1973 y 1978 (Hilbink, 2007). Antes de terminarse su periodo, el gobierno Aylwin también buscó implementar una ley estipulando que jueces especiales estuvieran a cargo de los procesos contra miembros de las Fuerzas Armadas y que sus procedimientos judiciales se llevaran a cabo en secreto (Hilbink, 2007). Este esfuerzo fue rechazado tanto por miembros de la izquierda como de la derecha política en Chile, ya sea por la cláusula referente a los procesos secretos como por divisiones internas frente al contenido de la ley.

A pesar de estos obstáculos y de la cercanía entre la rama judicial y las Fuerzas Armadas, el gobierno Frei logró mayores avances en materia de justicia, pero también otorgando concesiones importantes. En 1995, la Corte Suprema condenó al general retirado Manuel Contreras (jefe de la policía secreta del régimen militar) y a su segundo al mando, el general Pedro Espinoza, por el asesinato de Orlando Letelier (el ministro de Relaciones Exteriores durante el gobierno de Allende) en 1976. A raíz de su condena, el gobierno Frei pudo encarcelar a ambos oficiales. Sin embargo, este logro implicó que el gobierno Frei aprobara aumentos salariales para los oficiales y propuestas de legislación para dar clausura a seiscientos procesos judiciales pendientes contra miembros de las Fuerzas Armadas (Hilbrink, 2007).

En relación con el apoyo social y la apropiación local, uno de los factores fundamentales que le permitieron a las Fuerzas Armadas en Chile eludir las restricciones intrínsecas a un proceso de reforma del sector seguridad y reformulación de las relaciones cívico-militares fue el apoyo popular considerable que comandaron en el momento de la transición. Muchos chilenos, aunque críticos de los abusos de Derechos Humanos acaecidos durante el régimen militar, le otorgan al régimen el haber salvado al país del caos y encaminarlo hacia la prosperidad económica (Hunter, 1996, p. 29). Aunque insuficiente para alcanzar la victoria en el plebiscito de 1988, el $43 \%$ del voto a favor de la continuación de la dictadura de Augusto Pinochet evidencia el apoyo popular considerable del que disponía el régimen militar (Hunter, 1996, p. 29). De igual forma, encuestas de percepción posteriores continuaron registrando un grado sustancial de apoyo popular a las Fuerzas Armadas (Fontana, 1992). Ese 
apoyo fue instrumental para poder instaurar las prerrogativas institucionales y políticas que terminarían blindando a las Fuerzas Armadas frente a cualquier esfuerzo por reducir su fortaleza institucional, profesional o política en la democracia.

Para abordar la variable de economía politica, es importante aclarar que, como parte de los esfuerzos para preservar la autonomía militar del control democrático civil, el régimen militar tomó medidas para asegurar que las Fuerzas Armadas tuvieran amplitud de recursos fiscales. La Ley Orgánica de las Fuerzas Armadas de 1989 estipuló que el presupuesto de defensa no podía caer por debajo de su nivel absoluto de 1989, y se les aseguró a las Fuerzas Armadas un control del $10 \%$ de las utilidades de la Corporación Nacional del Cobre de Chile (Codelco), con un monto mínimo de 180 millones de dólares (Rojas, 1994). Sin embargo, la proporción de defensa en el presupuesto nacional se redujo de $11,34 \%$ a 8,65 \% en 1995, pues se destinó una mayor proporción del presupuesto a gasto social orientado a mejorar las condiciones de salud pública, educación, seguridad social, vivienda y trabajo. Así, en lugar de precipitarse incrementos súbitos en el presupuesto de defensa, el rubro aumentó de manera modesta, pasando de 401.744 a 439.007 millones de pesos entre 1989 y 1995 (Hunter, 1996, pp. 459-460). Por consiguiente, el gasto militar se ha preservado cerca de sus niveles durante el régimen militar, pero ha enfrentado competencia por recursos con políticas públicas orientadas a abordar necesidades socioeconómicas.

Por último, en lo que concierne a la dimensión internacional, la naturaleza controlada de la transición democrática chilena y la concentración de esfuerzos políticos en las batallas alrededor de la depuración del personal civil del sector seguridad, la implementación de las prerrogativas institucionales de las Fuerzas Armadas en la transición y la reforma constitucional produjeron un proceso contencioso y hermético, con poca participación de la sociedad internacional. En cuanto al papel internacional de las Fuerzas Armadas una vez se llevó a cabo la transición democrática en Chile, se puede aseverar que las Fuerzas han sido reacias a contribuir, particularmente en el campo de misiones internacionales de mantenimiento de la paz o estabilización, y, adicionalmente, conciben el espacio internacional primordialmente en términos de proyección de poder y realización de un destino manifiesto nacional. 
Las Fuerzas Armadas chilenas han enviado números limitados de efectivos a estas misiones, entre ellas a Camboya, El Salvador, India, Pakistán y el golfo Pérsico. De igual forma, en lugar de adquirir un papel activo como miembros de las misiones y tener un desempeño militar distintivo, las fuerzas chilenas involucradas han adoptado un rol pasivo de observadores (Hunter, 1996, p. 32). De igual forma, Chile rechazó el prospecto de mantener un contingente militar disponible cuando fuera necesario para misiones de mantenimiento de paz de la ONU, y también ha rechazado peticiones específicas por asistencia en forma de tropas ("Chile ha sido reticente a enviar personal...", 1995; "Esperan aumento de participación chilena...”, 1994; Hunter, 1996, pp. 32-33).

Desde 1989, la Armada chilena desarrolló el proyecto Mar Presencial. El plan consiste en expandir las pretensiones chilenas más allá de la zona económica exclusiva de doscientas millas náuticas, para incluir un área que se expande desde la Isla de Pascua hasta la Antártida, con el propósito de desarrollar la pesca oceánica, conducir investigación marina y científica, y construir un puerto en la Isla de Pascua para poder llevar a cabo ejercicios navales en el Pacífico Sur (Hunter, 1996, p. 33). Aunque la explotación económica y la exploración científica son incluidos como motivos del proyecto, el objetivo de proteger el poder y la soberanía chilena es también una motivación que parte de la ambición de proyectar poder. Simultáneamente, el proyecto rescata un elemento central del nacionalismo chileno: la idea de que el destino nacional de Chile depende de su dominio sobre el Pacífico del Sur (Hunter, 1996, p. 33).

\section{Reforma del sector seguridad en El Salvador y Guatemala (1960-1996)}

A diferencia de los casos analizados anteriormente, tanto en El Salvador como en Guatemala los procesos de reforma del sector seguridad fueron una consecuencia inmediata de los acuerdos de paz (Amaya, 2006; Call, 2002; Glebbeek, 2001; Stanley, 2006; Stanley \& Holiday, 2002). Una de las condiciones primordiales de los Acuerdos de Chapultepec fue la reforma del sector de seguridad, principalmente la transición hacia el control civil. Para cumplir 
este propósito, se consideró fundamental que se estableciera una nueva fuerza de policía controlada por las autoridades políticas civiles (Pérez, 2003). En paralelo, los Acuerdos de Chapultepec redujeron el número de efectivos de las fuerzas de seguridad de 60.000 a 6.000, y conminaron la desmovilización de la Guardia Nacional, dejando la Policía Nacional como el aparato de seguridad a cargo del orden público (Amaya, 2006, p. 118). En el caso guatemalteco, en septiembre de 1996 el gobierno de Álvaro Arzú y la Unidad Revolucionaria Nacional Guatemalteca (URNG) firmaron el Acuerdo de Paz Firme y Duradera, que proponía enmiendas constitucionales para despojar a las Fuerzas Militares de la responsabilidad de garantizar la seguridad doméstica y establecer una Policía Nacional Civil (PNC) (Stanley, 2006, pp. 124-123) ${ }^{14}$.

Tomando en cuenta la variable de efectividad, los procesos de reforma policial en El Salvador y Guatemala fracasaron en sus objetivos iniciales. En ambos casos, los procesos buscaban reducir el número de efectivos de las fuerzas de seguridad y, simultáneamente, tener una mayor atención e impacto sobre las necesidades de seguridad ciudadanas propias del posconflicto en ambos países. Sin embargo, los procesos suscitaron deterioros notorios en materia de prevención y reducción de la violencia y el delito.

Uno de los primeros objetivos de la reforma policial fue poder solventar las necesidades de seguridad ciudadana del país con una Fuerza Pública depurada. Aunque en las semanas siguientes a la firma de los acuerdos de paz los efectivos de las fuerzas de seguridad salvadoreñas se redujeron de 60.000 a 6.000, y se desmovilizaron numerosas fuerzas como la Guardia Nacional y la Policía de Aduanas, con lo cual la Policía Nacional quedó efectivamente como el único aparato doméstico de seguridad en operación, se registró un auge en el crimen y la violencia evidenciado por un incremento en los casos de homicidios en el país, que pasaron de 3.229 en 1992 a 9.000 en 1994 (Amaya, 2006, p. 118; Call, 2002, p. 5). La prevalencia de la violencia y el crimen continúa siendo un problema drástico en El Salvador al reportar tasas elevadas de homi-

14 Puntualmente, los Acuerdos incluían las siguientes provisiones: la expansión a 20.000 efectivos de la PNC hacia finales de 1999; reformas constitucionales para remover a las Fuerzas Militares de tareas de seguridad interna y transferir esa responsabilidad únicamente a la PNC; la implementación de leyes de seguridad pública y estructuras apoyadas por Minugua, y el compromiso de formar durante seis meses a los miembros de la PNC en la Academia de Policía (Glebbeek, 2001, pp. 437-438). 
cidios, luego se puede aseverar que la capacidad de la Policía salvadoreña de prevenir y reducir el crimen es un fracaso que ha perdurado de su proceso de reforma del sector seguridad.

Asimismo, otro de los objetivos primordiales del proceso de reforma policial en El Salvador fue la composición de una nueva Policía Nacional heterogénea. Con base en los acuerdos de paz, $80 \%$ de la nueva Policía Nacional tendría que ser compuesta por desmovilizados del Frente Farabundo Martí para la Liberación Nacional (FMLN) (20\%) y por nuevos reclutas civiles (60 \%) (Pérez, 2003, p. 630). Sin embargo, además de que la Policía Nacional no tuvo la capacidad de absorber suficientes reclutas para solventar las necesidades de seguridad del país en la transición al posconflicto, el $20 \%$ de sus miembros pertenecientes a los antiguos servicios de seguridad continuaron sus comportamientos antidemocráticos porque mantuvieron su entrenamiento arraigado en la represión política, en lugar de la investigación o la prevención del crimen (Cruz, 2006, pp. 154-155).

Al igual que el caso salvadoreńo, el proceso de reforma policial en Guatemala propuso reducir el número de efectivos y, simultáneamente, atender las necesidades de seguridad ciudadana en el posconflicto. Aunque Guatemala (a diferencia de El Salvador) registró una reducción momentánea en su tasa de homicidios entre 1997 y 1999, al tiempo que la victimización del delito pasó de 67 \% en 1996 a 42 \% en 1999 (Oficina de Naciones Unidas contra la Droga y el Delito [Unodc], 2007; Proyecto de Opinión Pública de América Latina, 2008), estas ganancias no fueron sostenidas. Durante el siglo XXI, las tasas de homicidio en Guatemala se han incrementado sustancialmente, a tal punto que inclusive ha llegado a ser el sexto país más violento del mundo (UNODC, 2007).

En ambos países, en materia de control democrático civil se pensó que era indispensable desmilitarizar las nuevas fuerzas policiales para que fuera posible ejercer un control democrático civil. Sin embargo, por razones distintas, tanto la Policía en El Salvador como su institución homóloga en Guatemala mantuvieron elementos autoritarios y lograron mantenerse al margen de controles democráticos institucionales. Como consecuencia, su involucramiento directo en la represión político continuó. 
El proceso de reforma policial en El Salvador propendió al control democrático civil de las fuerzas de seguridad a través de su desmilitarización. Con este propósito, se trató de configurar una nueva Policía Nacional con nuevos reclutas provenientes de la sociedad civil, como también elementos desmovilizados del FmLn. Sin embargo, las Fuerzas Militares intentaron activamente socavar este esfuerzo, ya que veían esta nueva fuerza policial como una amenaza para su habilidad de continuar desarrollando prácticas corruptas, mientras que la presencia de exmiembros del FMLN la percibían como una amenaza a la estabilidad del Estado salvadoreño y, por consiguiente, decidieron tomar control de las instalaciones donde se instalaría la nueva academia policial y retuvieron equipos básicos como carros, radios y armas (Call, 2002, p. 400; Cruz, 2006, pp. 154-155). José Miguel Cruz sostiene que la reforma policial en El Salvador fue viciada por una contaminación autoritaria y aporta como evidencia el hecho de que la Unidad Especial Investigativa y la Unidad Antinarcóticos fueron transferidas directamente a la nueva Policía Nacional, donde una porción significativa de efectivos continuaron implicándose en ejecuciones extrajudiciales con motivos políticos (Call, 2002, p. 401).

La reforma policial en Guatemala no logró el control democrático civil de las fuerzas de seguridad a través de la desmilitarización. En los tres años posteriores a la firma de los acuerdos de paz en Guatemala, el $73 \%$ de sus miembros correspondían a antiguos miembros de la Policía Nacional (Glebbeek, 2001, p. 438), lo cual implica que en sus filas abundaban efectivos sindicados de abusos y violaciones de Derechos Humanos. De igual forma, la "nueva" Policía guatemalteca no fue abierta a la participación de múltiples donantes internacionales en su proceso de reforma y capacitación, por lo cual resultó en un proceso de capacitación defectuoso que no logró cambiar la cultura de corrupción y represión política endémica, fuera de los controles democráticos institucionales (Stanley, 2006, p. 129).

En relación con las reformas de justicia y desarrollo, tanto en El Salvador como en Guatemala los fracasos en términos de la efectividad de los procesos de reforma del sector seguridad tuvieron un impacto directo sobre la inhabilidad de garantizar condiciones propicias para el desarrollo económico y humano. En ambos casos, el fracaso en prevenir y reducir el delito y la impunidad rampante 
por la falta de capacidades en la investigación y administración de justicia ha causado un gran perjuicio a la actividad económica, particularmente a través de la estabilidad jurídica necesaria para piedras angulares del desarrollo como el emprendimiento o el empleo juvenil (Sampó, 2013).

Frente al apoyo social y la apropiación local, en el caso de El Salvador, el proceso tuvo una confluencia importante de una variedad de actores de la sociedad civil. El pueblo salvadoreño exhibió niveles notables de apoyo al proceso de reforma como producto de los acuerdos de paz, y, además, actores importantes en el ámbito cultural como la iglesia Católica desempeñaron un papel fundamental de apoyo. En contraste, el proceso de reforma en Guatemala ha sido, como explica Vicenç Fisas, ampliamente criticado por considerarse un proceso de reforma con "excesivas aspiraciones" (Fisas, 2010, p. 12) poco ajustadas a demandas populares o a las necesidades en seguridad de la población, lo cual podría explicar la falta de refrendación de los mismos acuerdos de paz en el plebiscito de 1999.

En relación con la economía politica, la instauración de políticas de mercado caracterizadas por una reducción del gasto estatal, incluyendo aquel destinado a la seguridad, probaría ser otro elemento catalizador del escepticismo (en algunos casos hostilidad) de las Fuerzas Armadas frente a los procesos de reforma del sector seguridad. Tanto en El Salvador como en Guatemala la reducción de efectivos de las fuerzas policiales era un objetivo primordial de los procesos de reforma, pero, como explica Kincaid (2000), las limitaciones impuestas sobre redireccionar o incrementar el gasto público o la capacidad burocrática estatal constituyó un freno importante en los procesos de reforma de las instituciones de seguridad pública (p. 53), ya que las fuerzas policiales se preocuparon más por mantener prerrogativas institucionales y efectivos provenientes del régimen anterior, lo cual condujo a una continuación de culturas y prácticas corruptas, antidemocráticas y represivas.

En cuanto a la dimensión internacional, puntualmente, la participación de donantes y aliados internacionales, estos procesos de reforma del sector seguridad tuvieron una aproximación diametralmente opuesta. Mientras que en El Salvador se probó un modelo multilateral en el cual la OnU y la sociedad internacional tendrían un papel importante, en Guatemala se optó por endilgar la 
capacitación y reforma de la fuerza pública a un socio por convenio internacional, de tal manera que se mantuvo casi intacta la cultura militarizada de las fuerzas policiales.

El proceso de reforma policial de El Salvador tuvo mediación internacional sustancial. La ONU, con el acompañamiento de Estados Unidos, Canadá, Suecia y España, auspició proyectos de reforma y capacitación policial inmediatamente después de la firma de los acuerdos de paz en 1992 (Amaya, 2006, p. 34). En paralelo, la ONU estableció una misión de observación denominada Grupo de Observadores de la Organización de las Naciones Unidas en El Salvador (ONUSAL) para monitorear el progreso de las reformas y asegurarse de que observaran el cumplimiento de los pactos definidos en los acuerdos de paz (O’Neill, 2004, p. 49). Esta misión también incluyó un componente policial diferencial denominado Crvpol para monitorear la capacitación y la reforma policial.

En contraste, el acompañamiento internacional a la reforma policial en Guatemala fue reducido. En primer lugar, la misión de observación de las Naciones Unidas en Guatemala no tuvo un mandato para desplegar un componente policial diferencial en el país, lo cual se reflejó en la decisión de optar por una ruta bilateral para el desarrollo y entrenamiento de la Policía guatemalteca a través de un convenio con la Guardia Civil Española (Glebbeek, 2001, p. 433; Stanley, 2006, p. 126).

\section{Conflicto de intereses}

Los autores no reportan ningún conflicto potencial de interés en la realización de este capítulo.

\section{Agradecimientos}

La elaboración de esta investigación sobre la gobernanza y la reforma del sector seguridad y del presente capítulo no habrían sido posibles sin la financiación y apoyo incondicional de la Universidad Militar Nueva Granada, a la cual extendemos nuestros agradecimientos. 


\section{Financiación}

Este trabajo fue apoyado por el proyecto de investigación INV-EES 2975 titulado "El panorama del post conflicto en Colombia: Un acercamiento desde la gobernanza y la reforma del sector seguridad", financiado en su totalidad por la Universidad Militar Nueva Granada.

\section{Referencias}

Allan, A., \& Pollack, B. (1990). The chilean elections of 1989. Bulletin of Latin American Research, 9(1), 1-23. https://doi.org/10.2307/3338214

Amaya, E. A. (2006). Security Policies in El Salvador. 1992-2002. En J. Bailey \& L. Dammert (eds.), Public Security and Police Reform in the Americas (pp. 132-147). University of Pittsburgh Press.

Asociación pro-Derechos Humanos [Aprodeh] (1994). De la tierra brotó la verdad. Crimen e impunidad en el caso La Cantuta. Asociación Pro-Derechos Humanos.

Atria, R. (1994). El Consejo de Seguridad Nacional [Papel de Trabajo n. ${ }^{\circ} 34$ ]. Programa de Estudios Prospectivos.

Basombrío, C. (2004). Seguridad ciudadana y actuación del Estado: Análisis de tendencias de opinión pública. Instituto de Defensa Legal.

Bawden, J. R. (2016). The Pinochet Generation: The Chilean Military in the Twentieth Century. University of Alabama Press.

Berg, L. A. (2012). Guns, laws and politics: The political foundations of rule of law and security sector reform. Hague Journal on the Rule of Law, 4, 4-30. https://doi.org/10.1017/ S1876404512000036

Burt, J. M. (2006). "Quien habla es terrorista”: The political use of fear in Fujimori's Peru. Latin American Research Review, 41(3), 32-62.

Call, C. T. (2002). Assessing El Salvador's Transition from civil war to peace. En S. J. Stedman, D. Rothchild \& E. Consuens (eds.), Ending civil wars (pp. 383-420). Lynne Reinner Press.

Chile ha sido reticente a enviar personal de las FF. AA. al extranjero (1995). El Mercurio. https://digital.elmercurio.com

Collier, S., \& Sater, W. F. (1996). A history of Chile: 1808-1994. Cambridge University Press.

Comisión de la Verdad y Reconciliación [CvR]. (2003). Reporte final. http://www.cverdad.org.pe

Comisión Nacional de Verdad y Reconciliación. (1991). Informe de la Comisión Nacional de Verdad y Reconciliación. Estudios Públicos, 41. https:/www.cepchile.cl/cep/site/ artic/20160303/asocfile/20160303183807/rev41_verdad.pdf

Costa, G. (2006). Two steps forward, one and a half steps back: Police reform in Peru, 20012004. Civil Wars, 8(2), 215-230. https://doi.org/10.1080/13698240600877361 
Costa, G., \& Basombrío, C. (2005). Liderazgo civil en el Ministerio del Interior: Testimonio de una experiencia de reforma policial y gestión democrática de la seguridad en el Perú. Instituto de Estudios Peruanos.

Costa, G., \& Nield, R. (2007). La reforma policial en Perú. Revista Latinoamericana de Seguridad Ciudadana, 2, 112-126. https://doi.org/10.17141/urvio.2.2007

Cruz, J. M. (2006). Violence, citizen insecurity, and elite maneuvering in El Salvador. En J. Baily \& L. Dammert (eds.), Public security and police reform in the Americas (pp. 148-168). University of Pittsburgh Press.

Donais, T. (2009). Inclusion or exclusion? Local ownership and security sector reform. Studies in Social Justice, 3(1), 117-131.

Degregori, C. I., \& Rivera, C. (1993). Fuerzas Armadas, subversión y democracia: 19801993. Instituto de Estudios Peruanos.

Denney, L. (2011). Reducing poverty with teargas and batons: The security-development nexus in Sierra Leone. African Affairs, 110(439), 275-294. https://doi.org/10.1093/afraf/ adr004

Denney, L. (2013). Liberal chiefs or illiberal development? The challenge of engaging chiefs in DFID's Security Sector Reform Programme in Sierra Leone. Development Policy Review, 31(3), 5-25.

Ensalaco, M. (1994). In with the new, out with the old? Journal of Latin American Studies, 26, 409-429.

Esperan aumento de participación chilena en misiones de paz. (1994). El Mercurio. https:// digital.elmercurio.com

Fisas, V. (2010). Introducción a los procesos de paz. Quaderns de Construcció de Pau, 12, 1-22.

Fitch, J. S. (1986). Armies and politics in Latin America: 1975-1985. En A. F. Lowenthal \& S. J. Fitch (eds), Armies and politics in Latin America (pp. 26-57). Holmes \& Meier.

Fitch, J. S. (1998). The Armed Forces and democracy in Latin America. The Johns Hopkins University Press.

Fontana, A. (1992). Chile y Argentina: Percepciones acerca del rol de las Fuerzas Armadas. Fundación Simón Rodríguez.

Fuentes, C. A. (2002). Resisting change: Security sector reform in Chile. Security and Development, 2(1), 121-131.

Geisse, F., \& Ramírez Arrayas, J. A. (1989). La reforma constitucional. Centro de Estudios Sociales.

Glebbeek, M. L. (2001). Police Reform and the Peace Process in Guatemala: The Fifth Promotion of the National Civilian Police. Bulletin of Latin American Research, 20(4), 430-452. https://doi.org/10.1111/1470-9856.00021

Godoy Arcaya, O. (1999). La transición chilena a la democracia: Pactada. Estudios Politicos, 74, 79-106.

González, M. (2013). La Conjura: Los mil y un dias del golpe. Catalonia.

Gordon, E. (2014). Security sector reform, local ownership, and community engagement. International Journal of Security and Development, 3(1), 1-18. https://dx.doi. org/10.5334/sta.dx 
Graham, C. (1992). Peru's APRA: Politics, parties and the elusive quest for Democracy. Lynne Reinner Publishers.

Groner Krogstad, E. (2012). Security, development and force: Revisiting Police Reform in Sierra Leone. African Affairs, 111(443), 261-280. https://doi.org/10.1093/afraf/ads004

Hänggi, H., \& Scherrer, V. (2008). Towards an integrated security sector reform approach in UN Peace Operations. International Peacekeeping, 15(4), 486-500. https://doi. org/10.1080/13533310802396475

Heupel, M. (2012). Rule of law promotion and security sector reform: Common principles, common challenges. Hague Journal on the Rule of Law, 4, 158-175. https://doi. org/10.1017/S1876404512000097

Hilbink, L. (2007). Judges beyond politics in democracy and dictatorship: Lessons from Chile. Cambridge University Press.

Homel, P., \& Masson, N. (2016). Partnerships for human security in fragile contexts: Where community safety and security sector reform intersect. Australian Journal of International Affairs, 70(3), 311-327. https://dx.doi.org/10.1080/10357718.2015.1126803

Hunter, W. (1996). State and soldier in Latin America: Redefining the military's role in Argentina, Brazil, and Chile. Diane Publishing.

Hunter, W. (1997). Continuity or change? Civil-military relations in Democratic Argentina, Chile and Peru. Political Science Quarterly, 112(3), 453-475.

Jaskoski, M. (2013). Military politics \& democracy in the Andes. The Johns Hopkins University Press.

Jobet, J. C. (1987). Historia del Partido Socialista de Chile. Ediciones Documentas.

Kincaid, D. A. (2000). Demilitarization and security in El Salvador and Guatemala: Convergences of success and crisis. Journal of Interamerican Studies and World Affairs, 42(4), 39-58. https://doi.org/10.2307/166278

Mauceri, P. (1995). State reform, coalitions and the Neoliberal 'Autogolpe' in Peru. Latin American Research Review, 30(1), 7-38. https://doi.org/10.1007/BF02738987

Nove, A. (1986). Socialism, economics and development. Allen \& Unwin.

Oficina de Naciones Unidas de Drogas y Delito [Unodc]. (2007). Crime and development in Central America: Caught in the crossfire. LAPOP.

Oosterveld, W., \& Galand, R. (2012). Justice reform, security sector reform and local ownership. Hague Journal on the Rule of Law, 4, 194-209. https://doi.org/10.1017/ S1876404512000115

O'Neill, W. G. (2004). Police Reform and Human Rights. En Programa de las Naciones Unidas para el Desarrollo, The Human Rights Strengthening Programme (pp. 7-17). PNud.

Pérez, O. J. (2003). Democratic legitimacy and public insecurity: Crime and democracy in El Salvador and Guatemala. Political Science Quarterly, 118(4), 627-644. https://doi. org/10.1002/j.1538-165X.2003.tb00408.x

Petras, J. F., \& Morley, M. H. (1974). How Allende fell: A study in U.S.-Chilean relations. Spokesman Books. 
Philip, G. (2013). Nationalism and the Rise of Peru's General Velasco. Bulletin of Latin American Research, 32(3), 299-293. https://doi.org/10.1111/blar.12031

Proyecto de Opinión Pública de América Latina [LAPOp]. (2008). Corruption victimization by Police. Americas Barometer Insights, 3. https:/www.vanderbilt.edu/lapop/insights/ I0803en.pdf

Real Academia Española. (2019). Efectividad. https://dle.rae.es/?w=efectividad\&m=form

Riley, J., \& Schulz, M. (2016). Shifting norms: Development Policy and the role of security sector reform in advancing human security. Journal of Global South Studies, 33(1), 147-200.

Roberts, K. M. (1995). From the Barricades to the Ballot Box: Redemocratizacion and political realignment in the Chilean Left. Politics \& Society, 23, 495-519.

Rojas, F. (1994). Chile y el gasto militar. En F. Rojas (ed.), Gasto militar en América Latina (pp. 239-278). Facultad Latinoamericana de Ciencias Sociales.

Sagredo, R., \& Gazmuri, C. (eds.). (2005). Historia de la vida privada en Chile. Aguilar Chilena de Ediciones.

Sampó, C. (2013). Violencia en Centroamérica: Las Maras en El Salvador, Guatemala y Honduras. Estudios de Seguridad y Defensa, 2, 139-158.

Stanley, W. D. (2006). El Salvador: State-building before and after democratisation. 1980-95. Third World Quarterly, 27(1), 102-122.

Stanley, W. D. \& Holiday, D. (2002). Broad participation, diffuse responsibility: Peace implementation in Guatemala. En S. J. Stedman, D. Rothchild \& E. Consuens (eds.), Ending civil wars (pp. 421-462). Lynne Reinner Press.

Taylor, L. (2007). Politicians without parties and parties without politicians: The Foibles of the Peruvian Political Class 2000-2006. Bulletin of Latin American Research, 26(1), 1-23.

Varas, A. (1981). Fuerzas Armadas y gobierno militar: Corporativización y politización castrense en Chile. Flacso Chile.

Walter, R. J. (2010). Peru and the United States 1960-75: How their ambassadors managed foreign relations at a turbulent time. Penn State University Press.

Weeks, G. (2008). A preference for deference: Reforming the military's role in Argentina, Chile and Peru. Third World Quarterly, 29(1), 45-61.

Weyland, K. (1996). Neopopulism and Neoliberalism in Latin America: Unexpected affinities. Studies in Comparative International Development, 31(3), 3-31.

Weyland, K. (1997). 'Growth with Equity' in Chile's New Democracy? Latin American Research Review, 32, 37-67. 